Meta

Journal des tradlucteurs

Translators' Journal

\title{
Dans les coulisses
}

\section{Pierre Danis}

Volume 24, numéro 1, mars 1979

La traduction juridique

URI : https://id.erudit.org/iderudit/002455ar

DOI : https://doi.org/10.7202/002455ar

Aller au sommaire du numéro

Éditeur(s)

Les Presses de l'Université de Montréal

ISSN

0026-0452 (imprimé)

1492-1421 (numérique)

Découvrir la revue

Citer cet article

Danis, P. (1979). Dans les coulisses. Meta, 24(1), 124-129.

https://doi.org/10.7202/002455ar

Ce document est protégé par la loi sur le droit d'auteur. L'utilisation des services d'Érudit (y compris la reproduction) est assujettie à sa politique d'utilisation que vous pouvez consulter en ligne.

https://apropos.erudit.org/fr/usagers/politique-dutilisation/
Cet article est diffusé et préservé par Érudit.

Érudit est un consortium interuniversitaire sans but lucratif composé de l'Université de Montréal, l'Université Laval et l'Université du Québec à Montréal. Il a pour mission la promotion et la valorisation de la recherche. https://www.erudit.org/fr/ 


\section{Dans les coulisses}

Devant l'instrument diplomatique, paraphé et ratifié, peu de gens songent au rôle de la traduction et du traducteur. Il reste que l'un et l'autre interviennent dans l'élaboration de bon nombre des engagements internationaux coniractés aujourd'hui dans le monde. Déjà présente dans toutes les sphères de la vie nationale, la traduction joue dans l'action diplomatique canadienne un rôle méconnu mais essentiel. Aussi n'est-il pas inutile de jeter un peu de lumière sur son intervention dans les rapports bilatéraux et multilatéraux entre le Canada et la communauté des nations. Toutefois, comme je n'ai pas la prétention de couvrir la totalité d'un sujet si vaste, mes propos se limitent à la perspective et au rôle du Service des traductions détaché auprès du ministère des Affaires extérieures. Est-il nécessaire d'ajouter que les opinions et les observations qui suivent sont personnelles et, forcément, discrètes ?

D'abord et au risque de répéter un truisme, en diplomatie comme dans nombre d'autres domaines la forme est largement tributaire du fond. La langue est donc un instrument qui doit s'avérer souple et adaptable. A cet égard, il est coutumier de croire que le langage diplomatique est fait presque uniquement de formules consacrées. Certes, certains termes sont encadrés par l'usage, mais même des secteurs relativement «lents » comme les conventions protocolaires n'échappent pas à l'évolution. Les formules de politesse se décontractent quelque peu, tout comme ceux qui les utilisent. Par contre, tout le monde n'évolue pas au même rythme et le traducteur marche souvent sur des \& œufs diplomatiques 》, reconnus pour être très cassants. $\AA$ titre d'exemple, le mouvement de libération de la femme et, partant, du langage commence à soulever quelques problèmes, le fameux «Ms » notamment. "Madame », complément logique et commode de "Monsieur », n'a pas l'heur de plaire à tous, alors que Madel et Cie ont l'air farfelu de la nouveauté. Il y a eu à l'occasion des chassés-croisés téléphoniques sur cette épineuse question protocolaire qui, sans être capitale, exige cependant d'être résolue sous peine, sinon d'un incident, du moins d'un embarras diplomatique.

Par ailleurs, si le cérémonial diplomatique est généralement marqué au coin d'une étiquette étudiée, il ne faut pas croire pour un instant que la traduction se fait chez nous en habit et haut-de-forme. A l'instar de l'agent diplomatique, le traducteur doit, plus souvent qu'autrement, improviser avec bonheur 
faute de pouvoir devancer l'événement. Les conditions d'exécution sont la plupart du temps loin d'être idéales. Par exemple, il faut parfois compter avec des đétails aussi terre à terre que le décalage horaire et l'heure de mise sous presse. Il est gênant que la presse canadienne apprenne des services de nouvelles étrangers la signature par le Canada d'un accord bilatéral. Aussi ne faut-il pas se surprendre d'une facture quelque peu négligée à l'occasion. De telles conditions président à la traduction même d'instruments majeurs. Si le traducteur a eu, par un heureux hasard, le temps voulu pour faire le projet initial, il ne lui faudra pas s'étonner de voir arriver à $11 \mathrm{~h}$ une quarantaine de modifications proposées par l'autre partie, et sur lesquelles il lui faut statuer dans la demi-heure qui suit, ou d'être convoqué à $6 \mathrm{~h}$ du matin afin de traduire les dernières modifications négociées au cours de la nuit.

Aux problèmes d'intendance, il faut ajouter l'étendue des activités régies par le droit international. En effet, on peut dire de ce dernier qu'il est le prolongement à l'échelle supranationale des mécanismes nationaux de réglementation. Appelé à suivre l'évolution effrénée du $\mathrm{xx}^{e}$ siècle à l'instauration du régime de l'interdépendance, il doit forcément et continuellement repousser ses frontières. Pour un pays comme le nôtre, très ouvert sur l'extérieur, cela signifie gérer une gamme impressionnante de domaines avec des ressources humaines somme toute limitées. Membre de la communauté occidentale et surtout voisin de la première puissance commerciale et technologique du monde, le Canada est condamné à aller de l'avant. Il est d'ailleurs à l'avant-garde dans certains domaines du droit international, notamment en matière de garanties nucléaires et de droit de la mer, et dans de tels cas la jurisprudence établie ne peut, au mieux, que servir de guide dans l'élaboration et la formulation des concepts nouveaux. De surcroît, le Canada conserve une partie de la prééminence internationale dont il a hérité à l'issue de la Seconde Guerre mondiale et à ce titre intervient activement dans divers secteurs de la diplomatie multilatérale. Son activité au sein du système des Nations unies l'amène notamment à participer aux travaux de divers organismes et ainsi à être appelé à se prononcer aussi bien sur la forme que sur le fond. La Conférence sur le droit de la mer en constitue sans doute l'exemple le plus actuel puisque le Canada participe directement aux travaux du comité de rédaction du projet de Traité des océans.

Cette activité du Canada sur le plan international s'exerce toutefois dans le cadre d'un régime fédératif, ce qui ajoute parfois aux difficultés de traduction en ce sens que plus il y a d'intervenants plus il y a de points de vue tant sur la forme que sur le fond. S'il est tout à fait normal de procéder à des consultations fédérales-provinciales dans des dossiers où les responsabilités sont partagées, il n'en reste pas moins que des discussions de ce genre peuvent difficilement porter uniquement sur le fond. Compte tenu de la situation politique et linguistique au Canada, il n'est pas nécessaire de taxer son imagination pour concevoir que les sources de controverse, langagière à tout le moins, sont fécondes. Même si la traduction elle-même n'est pas soumise à l'examen, le seul fait du texte collectif où se juxtaposent souvent divers styles ajoute aux problèmes de traduction. Or, cette collectivisation de la rédaction est déjà présente 
au sein même de l'administration fédérale puisqu'il n'est pas rare qu'au gré des multiples étapes de la consultation et de l'agrément, diverses contributions soient apportées au puzzle que devient à l'occasion le projet d'accord. Il n'est pas étonnant que le traducteur trouve souvent non pas son fil d'Ariane mais un écheveau à démêler.

De surcroît, le fait de deux langues officielles signifie que pour un instrument bilatéral il n'est pas rare de se retrouver en présence de trois textes qui font foi et qui sont confrontés minutieusement. Il arrive même que chaque version soumise soit retraduite par l'autre partie afin d'en vérifier l'exactitude. Sachant comment il peut être difficile de s'entendre sur un document unilingue, il n'est pas exagéré de s'attendre à des difficultés d'ajustement, surtout si des langues et des civilisations très différentes sont en jeu. Ainsi, il surgit généralement plus de « « différends formels» avec un pays ayant un vécu culturel et linguistique tout à fait différent du nôtre. Certains sont alors tentés d'opter pour une traduction littérale. C'est ainsi qu'on voudra traduire «along the lines » par le «long de la ligne » quand il s'agit de s'inspirer d'un accord existant. Certes, l'exemple est extrême, mais il est représentatif d'une prudence excessive que provoque souvent chez le non-initié la présence de plusieurs textes faisant foi. Le littéralisme procède également des démarches juridiques différentes de l'esprit anglo-saxon et de l'esprit français, l'un énumératif, l'autre synthétique.

Le fait que l'anglais et le français soient les deux grandes langues de la diplomatie devrait faciliter l'égalité de statut. Le phénomène n'a cependant pas que des conséquences bénéfiques pour le traducteur. Il peut être difficile de s'affranchir des précédents, le voisinage des deux langues ajoutant d'ailleurs à la subtilité de la différenciation. S'il est facile de débouter le diplomate étranger qui propose de traduire «along the lines» par « le long de la ligne», il est parfois moins facile de convaincre le diplomate canadien, anglophone ou francophone, de l'à-propos d'une traduction moins littérale qui s'écarte à la fois de traductions antérieures et de l'original. L'enjeu est encore plus risqué si les négociations sont difficiles et qu'on ne tient pas à indisposer l'autre partie pour une simple question de forme. D'ailleurs, la langue de référence est la langue dans laquelle a été négocié l'accord et il est de tradition que celle-ci soit plus égale que les autres. On s'attend donc que le traducteur ajuste son texte sur loriginal et s'il a le malheur de vouloir éviter une répétition il risque fort d'être rappelé à l'ordre. Il reste que la. Loi sur les langues officielles, en donnant un statut légal au français et à l'anglais, a mis en branle un processus d'ajustement qui devra aboutir au respect intégral des deux langues sans l'asservissement de l'une ou de l'autre.

A cet égard, il existe encore trop souvent une présomption d'infériorité à l'égard du français «canadien ». S'il existe un modèle international ou étranger, le traducteur aura beaucoup de mal à faire accepter une autre version par son client. Ce prestige, qui n'est pas toujours mérité, oblige souvent à ne pas sortir de l'ornière. Ainsi, si l'ONU parle de "contrôle » des armements ou d' « établissement de la paix $»$, on passera difficilement au traducteur la « maîtrise \$ ou la « pacification ». Si l'OCDE parle de «bénéficiaire effectif » pour 
rendre « beneficially owned », il n'y a rien à faire. Il y a lieu de signaler, toutefois, un "dégel » qui tient, pour l'essentiel, à l'établissement de meilleures communications entre les agents du Ministère et les traducteurs.

Face aux velléités autonomistes du traducteur, l'agent francophone faisait traditionnellement office de contrôleur des textes traduits. $\grave{A}$ cet égard, comme presque toujours en traduction, l'insuffisance de la consultation et de la concertation est à l'origine des principales difficultés. Par exemple, le protocole devient un mémoire sous la plume d'un agent qui ne sent aucunement le besoin d'une confirmation. Le mouvement amorcé depuis quelques années vise à instaurer la collaboration, tout en reconnaissant au Ministère l'ultime responsabilité du texte. Cette notion n'est toutefois pas encore entrée dans les mœurs et s'il y a un nombre croissant de fonctionnaires qui nous consultent, cela reste un choix individuel et non une habitude collective. Malgré cette carence, la réceptivité à la notion de dualité langagière est fort bonne, si ce n'est du seul fait des séjours que font nos diplomates dans des pays étrangers et de l'obligation concomitante de s'initier à diverses langues. Ce même phénomène fait cependant de certains de nos diplomates des clients exigeants.

Face à ces derniers le traducteur des Affaires extérieures se doit de posséder un bagage impressionnant de qualités. Dans l'idéal, c'est une personne polyvalente, dotée d'une plume bonne et rapide, d'un esprit à la fois rigoureux et éclectique et douée d'une grande capacité de travail. Elle doit suivre de près l'actualité nationale et internationale et posséder la faculté de synthèse lui permettant de faire le lien entre les grands dossiers bilatéraux et multilatéraux et les instruments diplomatiques qui en sont souvent l'aboutissement. $\AA$ l'instar de ses clients, elle doit savoir doser tact et fermeté. Mais comment en arriver là ?

La formation générale du traducteur et, à plus forte raison, sa spécialisation restent des sujets fort controversés. Dans le cas qui nous occupe, devonsnous spécialiser le traducteur en droit international ou initier des juristes à la traduction? Il faut dire d'abord que le recrutement dans un secteur aussi spécialisé n'est pas chose facile au Canada. Cette solution serait sans doute la meilleure, à supposer que l'on puisse trouver des disciples de Thémis sensibilisés aux questions de langue et intéressés à faire de la traduction. A l'évidence, le problème du niveau collectif de la langue française au Canada : se pose aussi pour la langue juridique. . Le rôle accessoire qui semble réservé à la tenue langagière dans les écoles de droit reflète bien le milieu, mais prépare assez mal à la traduction.

En raison même des difficultés de recritement, la plupart des traducteurs œuvrant dans le domaine s'y sont initiés par la pratique et, somme toute, les résultats n'ont pas été si mauvais. D'aucuns prétendraient qu'ils ont été fort satisfaisants. D'autre part, il est indéniable qu'un complément de formation théorique serait bénéfique aux praticiens, car l'approche ponctuelle ne peut suffire. Il faudrait cependant que les universités offrent au traducteur une formation davantage adaptée à ses besoins, ce qui n'est pas chose facile en raison de la clientèle restreinte dans le domaine. Il n'est pas du tout impossible qu'une for- 
mule mixte de recrutement et de formation s'avère être la seule solution viable. Dans une telle optique, les spécialistes pourraient être appelés à jouer le rôle de personnes ressources; conseillers et contrôleurs, ils travailleraient de concert avec une équipe de «demi-spécialistes ».

D'ailleurs, une telle formule aurait l'avantage d'assurer encore plus la polyvalence indispensable au traducteur cuvrant en droit international. Ce dernier, à l'instar de l'avocat « de province», ne peut se payer le luxe d'une double spécialisation. Il doit donc tenter de suivre l'évolution dans toutes les branches du droit. Le temps à investir est donc considérable et le défi de taille, surtout si l'on considère la complexité technique de secteurs comme la coopération nucléaire ou les télécommunications par satellites. Il n'est pas donné à tout le monde de connaître les cuves anticriticité ou les satellites sur orbite géosynchrone. Et à supposer que les spécialistes du domaine puissent lui foumir les réponses voulues, il lui faut quand même les insérer dans le cadre juridique approprié.

À l'évidence, le traducteur est appelé à consulter des spécialistes, aussi bien dans le domaine juridique que dans le domaine visé par l'instrument qu'il doit traduire. Cette collaboration constitue d'ailleurs une des facettes les plus souvent ignorées de la traduction, qu'elle soit de nature juridique ou autre. Le traducteur stéréotypé, à peine visible derrière l'amas de dictionnaires et d'ouvrages de référence, ne correspond pas toujours à la réalité. Certes, la recherche est un des éléments indispensables de l'acte professionnel, mais on doit l'entendre dans un sens large et savoir utiliser au maximum les ressources, notamment humaines, à sa disposition. L'optimisation d'un tel réseau suppose évidemment des talents de communicateur qu'on n'associe pas toujours avec les qualités du traducteur.

Cette aptitude à communiquer est particulièrement critique dans un milieu comme celui des Affaires extérieures et ce, en raison du mandat et du mode de fonctionnement du ministère. Appelé à gérer les relations extérieures du Canada et, partant, les prolongements à l'étranger des activités nationales, le ministère mène de front un nombre impressionnant de dossiers. La complexité du réseau est donc, au départ, fort considérable puisque celui-ci déborde largement le cadre administratif des Affaires extérieures. De surcroît, le régime de la permutation permanente pratiqué par le ministère signifie que le traducteur traite continuellement avec des clients nouveaux. Outre les indispensables contacts à refaire, il arrive souvent que des renseignements fondamentaux partent avec le titulaire. Et allez donc téléphoner en Australie pour faire éclaircir le sens d'une phrase! Le traducteur doit donc très bien connaître la filière officielle et officieuse.

Dans cette optique, il est évidemment souhaitable que le traducteur soit le maître d'œuvre de ses relations avec le client. Pour cela il lui faut être authentiquement professionnel et en mesure de mener son travail du début à la fin. C'est vers cet objectif que tend le service pour l'ensemble de ses prestations et plus particulièrement pour les textes dont la portée ou la diffusion commandent une qualité supérieure et dônt la réalisation exige une liaison étroite avec le 
client. Les instruments diplomatiques entrent évidemment dans cette catégorie. Un tel objectif suppose continuité et stabilité, deux ingrédients fort rares dans le milieu de la traduction ces temps-ci. Il faudra cependant en revenir à un meilleur équilibre si nous voulons que les traducteurs obtiennent la reconnaissance capitale, celle de leur compétence.

Ce rapide survol du rôle que joue le Service de traduction des Affaires extérieures n'a évidemment pas la prétention d'une étude exhaustive du mandat actuel ou éventuel de la traduction dans la diplomatie canadienne. Il se veut par contre une démystification et aussi une valorisation de la traduction et du traducteur. Une démystification parce que le prestige rattaché aux affaires internationales fait trop facilement oublier les \& servitudes》 de la traduction et une valorisation parce que la traduction est effectivement partie intégrante de la réalité diplomatique canadienne. Le traducteur, s'il n'est pas appelé à jouer les premiers rôles, peut à tout le moins tirer satisfaction d'avoir bien manceuvré dans les coulisses.

Pierre Danis 